\title{
Infectious disease outbreaks highlight gender inequity
}

\author{
Here the implications of gender inequity in the face of infectious disease outbreaks such as Ebola and SARS-CoV-2 \\ in Africa are discussed, with a proposal as to how we can address the unequal burden of outbreaks on women.
}

\author{
Agnes Binagwaho and Kedest Mathewos
}

$\square$ ender inequity poses significant obstacles to the improvement of the wellbeing of women and exacerbates the burden that women bear during emergency health crises, such as infectious disease outbreaks. The 20132016 Ebola virus disease (EVD) and the current SARS-CoV-2 pandemic are prime illustrations of the implications of gender inequity in the face of such outbreaks. The 2013-2016 Ebola outbreak infected 28,625 people and killed 11,325 people in Africa $^{1}$. The COVID-19 pandemic, as of 25 January 2022, has caused more than 358 million infections and 5.62 million deaths, with 10.63 million of the infections and 236,399 of the deaths occurring in Africa ${ }^{2}$. Examining the impacts of these infectious diseases according to sex reveals a clear imbalance, with a much higher vulnerability in women than men when it comes to the indirect health, social and economic consequences of such health crises in Africa. Understanding why this is the case is needed to underpin policies to reduce the disproportionate burden of infectious diseases by sex in future. Note, however, that this phenomenon is not unique to Africa but is widespread across the globe.

\section{Incidence and fatality rates}

Various studies have examined Ebola infection and fatality rates disaggregated by sex and most have found a difference of a few percentage points in the proportion of female and male Ebola virus cases, with women bearing the larger share of disease. A study carried out by the World Health Organization (WHO) Ebola response team reported that of 20,035 confirmed and probable cases of EVD between December 2013 and 11 August 2015, 48.8\% of the cases were in men, but this difference was not statistically significant ${ }^{3}$. Moreover, the percentage of male cases in three of the most affected countries - Guinea, Sierra Leone and Liberia - did not differ significantly from the percentage of males in the general population except for the Gueckedou district in Guinea, where $63.4 \%$ of EVD cases were male. This study also reported that the risk of exposure was higher for women, whereas the fatality rate once infected was higher for men.

While we are still in the midst of the COVID-19 pandemic, in Africa, the incidence and case fatality rate of SARS-CoV-2 infection in women is lower than in men $^{4}$. The greater number of deaths among men could potentially be attributed to the higher prevalence of chronic illnesses. Note that the lack of sex-disaggregated data continues to be a challenge, although this has improved over the course of the pandemic. Global Health 50/50 estimates that, as of mid-October 2021 , only $60 \%$ of cases and $70 \%$ of deaths globally have been sex disaggregated ${ }^{5}$.

While gender comparisons in infection and mortality rates for Ebola and COVID19 have not been accurately determined due to a lack of consistent and adequate-quality sex-disaggregated data, there is clear evidence of gender inequality in the indirect health, social and economic impacts of infectious disease outbreaks.

\section{Outbreaks disrupt health systems}

Infectious disease outbreaks occur in societies that are already gender inequitable. Indirect consequences of infectious disease - such as the interruption of essential health services, the slowdown of the economy and lockdowns - exacerbate existing inequities in society. During the outbreaks of both Ebola and COVID19 , the interruption of maternal health services, with barriers on both the supply and demand side, significantly hampered progress towards improving maternal health outcomes $^{6,7}$. Fear, mistrust and scarcity of health system resources and public health measures to prevent the spread of these infectious diseases, as well as lack of community education, contributed to a decline in the utilization of maternal health services. Again, despite global knowledge of the potential contributors to health system interruption in case of outbreaks, the lack of resilient health systems resulted in an increase of deaths due to diseases unrelated to outbreak infections. This lack of resilience disproportionately threatens the health and wellbeing of women, because fewer strategies and systems are present to protect them. For instance, maternal deaths increased in the three West African countries most affected during the EVD outbreak $^{6}$, and now data reveal a similar increase during the COVID-19 pandemic ${ }^{7}$. Maternal health outcomes, which serve as a core indicator of the functionality of a health system, are often threatened with the emergence of any new health crisis.

\section{Gender-based violence and economic consequences}

Beyond maternal health outcomes, public health measures implemented to curb the spread of pathogens disregard the disproportionate negative impact of these measures on girls and women. For instance, lockdowns and movement restrictions resulted in a rise in the risk of gender-based violence during the Ebola outbreak $^{8}$. Despite this knowledge, the implementation of lockdowns during the COVID-19 pandemic was not, in the majority of countries, coupled with adequate pre-emptive strategies to address this risk $^{8}$. Instead, gender-based violence during COVID-19 has been named the 'shadow pandemic' across the world, with, for example, a report of a $48 \%$ increase in gender-based violence cases by ministries responsible for gender in the East African region'. Similarly, the slowdown of the economy that accompanies infectious disease outbreaks further affects the economic wellbeing of women. For instance, women are overrepresented in the informal sector, which was particularly hit during this pandemic as a result of the public health measures put in place to curb the spread of the virus ${ }^{4}$. Thus, it is clear that Ebola and COVID-19 strengthened the cycle of infectious disease, poverty and structural violence, thereby contributing to a more gender-inequitable world. 


\section{Gender disparities in risk to healthcare providers}

The fact that women are severely underrepresented in leadership roles in any sector is not news. Seventy per cent of the global health workforce is composed of women but only $25 \%$ are in leadership positions ${ }^{10}$, suggesting that women have a reduced influence in health sector management. It has been proven that there are significant benefits to having women make decisions, because they are more likely to confront and address issues that disproportionately affect the vulnerable, including women and children ${ }^{11}$. Moreover, female leaders are likely to have a more participatory leadership style than that men $^{12}$. The gender imbalance in global health leadership fuels a vicious cycle of gender inequity because having fewer women in leadership positions stands as a barrier to the improvement of women's status in society, which in turn leads to few women in decision-making positions.

As a result of their overrepresentation in positions of health service delivery, women are providing the majority of care but have little say in the decisions on health service delivery. That women are overrepresented in nursing, community health worker, cleaning and laundry professions is an indication that they provide the majority of frontline care work in community and hospital settings and have more exposure to pathogens ${ }^{13}$. Moreover, despite constant exposure to SARS-CoV-2 in frontline healthcare roles, many female workers have not been provided with necessary protective equipment. For example, the design of one-size-fits-all personal protective equipment (PPE) fails to consider the female body. PPE is designed to fit an average man, forcing female healthcare workers to wear ill-fitting PPE that could increase their exposure to the SARS-CoV-2 and ultimately may increase their vulnerability ${ }^{14}$.

While female health professionals in general face greater exposure risk to infectious diseases, this risk is far worse in Africa. Weak health systems that lack the necessary medical drugs and equipment, as well as accessible, fully equipped health facilities, undermined the ability of African health professionals in Guinea, Liberia and
Sierra Leone to rapidly respond to the virus outbreak and treat those that were infected while protecting themselves from exposure ${ }^{15}$. On the other hand, only four patients were infected on US soil - indicating the capacity of the country to prevent the spread of the virus to the US population and their healthcare workers ${ }^{1}$. This difference in health system capacity, coupled with the fact that frontline healthcare workers are disproportionately female, means that in low- and middle-income countries, where the majority of Black health professionals work, health systems are not sufficiently equipped to protect their wellbeing.

\section{Inequity in prevention and treatment} Early in both outbreaks, the maternal and fetal outcomes of both the Ebola virus and the coronavirus were not known, making the effective and safe treatment of pregnant women and their fetuses difficult ${ }^{16,17}$. Moreover, even in the event of the discovery of a life-saving medical intervention, its use by pregnant women is delayed. Traditionally, it is considered unethical to include pregnant women in clinical trials due to their increased vulnerability and the assumption that certain characteristics of pregnancy might affect the medical conditions under study ${ }^{18}$. Therefore, all Ebola drug and vaccine trials conducted between 2013 and 2016 in Guinea, Liberia and Sierra Leone excluded pregnant women $^{16}$. During COVID-19, a study that evaluated the 927 COVID-19-related clinical trials in the WHO's International Clinical Trials Registry on 7 April 2020 found that $52 \%$ of the studies explicitly excluded pregnancy ${ }^{17}$. Although it is important to protect pregnant women against unnecessary risks, their exclusion should be avoided in cases where the existing knowledge gap on the safety and efficacy of drugs and vaccines is more harmful than the risk of inclusion as it delays pregnant women's access to life-saving care.

\section{Outlook}

We know two things for certain. First, gender inequity is not new and will continue to impact the wellbeing and livelihood of women if it remains unaddressed. Second, we will continue to face infectious disease outbreaks, in every country around the world and not only in Africa.

As a society, to pre-empt the challenges that women will face during the next public health threat, we need to prepare our health systems and communities to address them by using the lessons that we learn from gender inequities highlighted by prior outbreaks and pandemics. We need to first acknowledge that these challenges exist. Then we need to act to improve the systems that collect accurate sex-disaggregated data, and create resilient health systems capable of continuing the provision of ordinary care during health crises.

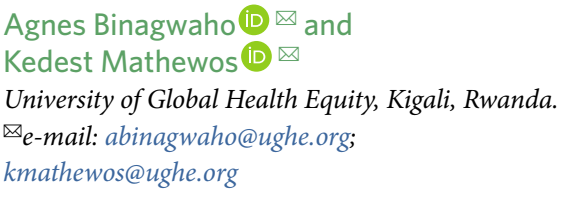

Published online: 4 March 2022

https://doi.org/10.1038/s41564-022-01075-2

References

1. Ebola Outbreak 2014-2016 - West Africa (WHO, accessed 16 November 2021); https://go.nature.com/3GqYWO1

2. COVID-19 Data Explorer (Our World in Data, accessed 16 November 2021); https://go.nature.com/3otCNs9

3. WHO Ebola Response Team et al. N. Engl. J. Med. 374, 96-98 (2016).

4. Fewer COVID-19 cases among women in Africa: WHO analysis. World Health Organization (4 March 2021); https://go.nature. com $/ 3 \mathrm{~s} l \times 74 \mathrm{H}$

5. The COVID-19 Sex-Disaggregated Data Tracker: October Update Report (Global Health 50/50, 2021); https://go.nature. com/3Lh6PsG

6. Jones, S. A. et al. BMJ Glob. Health 1, e000065 (2016).

7. Chmielewska, B. et al. Lancet Glob. Health 9, e759-e772 (2021).

8. John, N. et al. Dev. World Bioeth. 20, 65-68 (2020).

9. Gender-Based Violence in Africa During the COVID-19 Pandemic (UN Women, 2020); https://go.nature.com/3HwPr16

10. Batson, A., Gupta, G. R. \& Barry, M. Ann. Glob. Health 87, 67 (2021).

11. Downs, J. A. et al. Acad. Med. 89, 1103-1107 (2014).

12. Women in the Workplace 2021 (McKinsey, 2021); https:// go.nature.com $/ 34 \mathrm{DFwbe}$

13. Socio-Economic Impact of Ebola Virus Disease in West African Countries: A Call for National and Regional Containment Recovery and Prevention (UNDG, 2015); https://go.nature. com/3Lr9tfV

14. Chakladar, A. \& Ascott, A. Personal protective equipment is sexist. BMJ Opinion (9 March 2021); https://go.nature. com/3skJILV

15. Shoman, H., Karafillakis, E. \& Rawaf, S. J. Glob. Health. 13, 1 (2017)

16. Gomes, M. F. et al. Reprod. Health 14, 172 (2017).

17. Smith, D. D. et al. Am. J. Perinatol. 37, 792 (2020).

18. Kaye, D. K. Philos. Ethics Humanit. Med. 14, 11 (2019).

Competing interests

The authors declare no competing interests. 